Aung and Htay conclude that vasopressin shows neither harm nor any apparent advantage over epinephrine in the treatment of cardiac arrest. They propose, therefore, that the drug should not be recommended for use in this setting unless clear evidence becomes available to show its superiority to epinephrine.

Original article Aung K and Htay T (2005) Vasopressin for cardiac arrest: a systematic review and meta-analysis. Arch Intern Med 165: 17-24

\section{The obesity paradox in heart failure}

Contrary to the observation that excess body weight increases the risk of adverse health outcomes in the general population, there is some evidence to suggest that overweight and obese individuals with chronic disease are at lower risk than those of a 'healthy' weight. In their recent study, Curtis and colleagues have shown that this so-called obesity paradox also applies to patients with established heart failure.

By use of data from the Digitalis Investigation Group trial, the authors examined the relationship between BMI and outcomes in 7,767 patients with stable heart failure. During the mean follow-up of 37 months, survival rates were higher in those with higher $\mathrm{BMI}$ than in leaner patients. Multivariate analysis showed that higher BMI values were independently associated with a lower risk of cardiovascular death, death due to worsening heart failure and death from any cause. Underweight patients showed an increased risk of death, but this difference was not statistically significant.

Concluding that overweight or obese heart failure patients were at decreased risk of death compared with those of a 'healthy' weight, Curtis et al. speculate as to the mechanisms underlying this paradox. Heavier patients might be diagnosed earlier on in the disease process and might have less severe myocardial dysfunction than those with $\mathrm{BMI}$ values in the normal range. Alternatively, cardiac cachexia-a wasting syndrome associated with an increased risk of adverse outcomes-is likely to be more common in leaner patients. In addition, the study was based on a single measurement of BMI and so did not take into account the effects of weight loss or gain. The authors call for clinical trials to further investigate the obesity paradox in this setting.

Original article Curtis JP et al. (2005) The obesity paradox: body mass index and outcomes in patients with heart failure. Arch Intern Med 165: 55-61

\section{Dangers of aspirin withdrawal}

Aspirin is widely used in the secondary prevention of coronary events. A recent study by Ferrari and colleagues indicates that its withdrawal increases the risk of a new coronary event. This finding adds more evidence to the argument that withdrawal of aspirin before some surgical procedures might not be advisable.

Of a group of 1,236 patients hospitalized with acute coronary syndrome, 383 were known coronary disease patients. The new coronary event had occurred less than 1 month following aspirin withdrawal in 51 cases; thus aspirin withdrawal preceded coronary events in $4 \%$ of patients in the group as a whole and in $13 \%$ of those with pre-existing coronary disease. ST-segment elevation acute coronary syndrome was significantly more common among patients who stopped taking aspirin than in those who continued, and thrombosis of an uncoated stent occurred in 10 cases. Aspirin had been withdrawn because of patient non-compliance in 20 cases; minor surgery, fibroscopy, dental treatment and bleeding accounted for the remainder.

Ferrari et al. conclude that cessation of aspirin poses a significant risk in some patients with known coronary disease, and that the risk of late stent thrombosis was particularly high. It will be important to identify which patients are at risk and to educate patients, dentists and surgeons about the potential dangers of aspirin withdrawal.

Original article Ferrari E et al. (2005) Coronary syndromes following aspirin withdrawal: a special risk for late stent thrombosis. J Am Coll Cardiol 45: 456-459

\section{The 'visual stethoscope': portable ultrasound in the outpatient clinic}

A portable ultrasound device is useful in the initial assessment of patients referred to the outpatient cardiology clinic, according to a new 